\title{
Methods of Venous Thromboembolic Prophylaxis in Orthopedic Surgeries
}

\author{
Sheren A. Khalil, Ahmed S. Rizk, Ahmed A. Elsheikh, Bishoy N. Ragheb
}

a

Department of orthopedic surgery, Benha faculty of medicine ,Benha University, Egypt.

Correspondence to:

Bishoy N. Ragheb Department of orthopedic surgery, Benha faculty of medicine, Benha University, Egypt

Email:

bishoynabilbenha@gmail.com

Received: 3 June, 2020

Accepted :31 August, 2020

\begin{abstract}
:
Background: Venous thromboembolic prophylaxis after major orthopaedic surgeries is an essential topic to review and has a lot of controversies and differences regarding which method to emphasize and the duration of each method of prophylaxis to be used in daily practice in orthopaedic surgery. Here we mentioned the review of literature with respect to thromboembolic prophylaxis in orthopaedic surgery including the latest guidelines and we made a meta-analysis of data from many studies regarding the use of different pharmacological agents after major orthopaedic surgeries like total hip replacement (THR), total knee replacement (TKR), hip fracture and knee arthroscopic surgery. Method: we searched Medline via PubMed, SCOPUS, Web of Science, Cochrane Central Register of Controlled Trials (CENTRAL), and Google Scholar from 2010 till November 2019. The search retrieved 2089 unique records. We then retained 57 potentially eligible records for full-texts screening. Finally, 29 studies were included. Results: the rate of DVT was higher with DTI than with LMWH and lowest with FXaI, while the rate of PE was higher with DTI than FXaI and lowest with LMWH. The rate of major bleeding was higher with LMWH than with FXaI and lowest with DTI, indicating that FXaI has the lead in thromboembolic prophylaxis after THR or TKR with lower risk of bleeding compared to LMWH. Conclusion: FXaI was the most
\end{abstract} effective agent after THR and TKR. In hip fracture surgery and Knee arthroscopy, thromboprophylaxis is needed, but variable results regarding the drug choice warrant more research.

Keywords: Thromboembolic; prophylaxis; orthopedic 


\section{Introduction}

Venous thromboembolism (VTE) is a common complication during and after hospitalization for medical and surgical patients, including orthopaedic patients. More than half of all hospitalized patients are at risk for VTE, with a higher risk in surgical patients than in medical patients (1).

However, the overall VTE rates are in the range of $13 \%$ to $70 \%$, implying a large variability between institutions and countries. Without any prophylaxis, pulmonary embolism (PE) is responsible for 5\% to $10 \%$ of deaths in hospitalized patients. The incidence of fatal PE in hospitalized patients is $0.1 \%$ to $0.8 \%$ after elective general surgery, $2 \%$ to $3 \%$ after elective hip replacement and $4 \%$ to $7 \%$ after hip fracture surgery. Similarly, deep venous thrombosis (DVT) affects approximately $0.1 \%$ of persons per year (2).

The overall incidence of DVT in medical and general surgery hospitalized patients is in the range of $10 \%$ to $40 \%$; in comparison, the incidence of DVT ranges up to $40 \%$ to $60 \%$ in major orthopaedic surgery (3).

The overall average age- and sex-adjusted annual incidence of venous thromboembolism (VTE) is $0.117 \%$ (DVT, $0.048 \%$; PE, $0.069 \%$ ), with higher ageadjusted rates among males than females (0.130 vs $0.110 \%$, respectively). Both sexes are equally afflicted by a first VTE, men having a higher risk of recurrent thrombosis. DVT is predominantly a disease of the elderly with an incidence that rises markedly with age (4).

Death within one month of diagnosis occurs in approximately $6 \%$ of DVT patients and approximately $12 \%$ of PE patients. The cumulative ten-year incidence of recurrent VTE reaches $39.9 \%$ (35.4\% to $44.4 \%$ ) (2).

The incidence of VTE is low in children. Annual incidences of 0.0007 to $0.0014 \%$ children and $0.053 \%$ hospital admissions have been reported in Caucasian studies. This low incidence may be due to decreased capacity to generate thrombin, increased capacity of alpha-2-macroglobulin to inhibit thrombin, and enhanced antithrombin potential of vessel walls. The highest incidence in childhood is during the neonatal period, followed by another peak in adolescence. The incidence rate is comparatively higher in adolescent females because of pregnancy and use of oral contraceptive agents (5).

Pregnant women have a much higher risk of VTE than non-pregnant women of similar age and the risk has been shown to be higher after cesarean section than after vaginal delivery (6).

Orthopaedic patients are at higher risk among all patients for DVT and VTE. In the early 
2000s, despite the existence of VTE prophylaxis guidelines, the use of VTE prophylaxis was low (7). Currently, the adherence to the different VTE prophylaxis guidelines, especially for orthopaedic patients, is increasing.

We aimed at investigating the recent trends of research in the last ten years. There have been a tremendous advances in venous thromboembolism (VTE) prophylaxis. Guidelines have also been developed and put in action. We aimed at studying the current state of art in VTE prophylaxis after major orthopaedic surgeries.

\section{Materials and methods}

We performed this systematic review and meta-analysis in accordance to the recommendations of the Preferred Reporting Items for Systematic Reviews and MetaAnalyses (PRISMA) statement and Metaanalysis Of Observational Studies in Epidemiology (MOOSE) statement. PRISMA and MOOSE are reporting checklists for authors, editors, and reviewers of metaanalyses of interventional and observational studies. According to International committee of medical journal association (ICJME), reviewers must report their findings according to each of the items listed in those checklists (8).

The present review included studies that fulfilled the following criteria:
1- Studies that included adult patients who underwent one of the following procedures; hip fracture surgery, knee arthroscopy, total knee replacement, and total hip replacement.

2- Studies that assessed the safety and effectiveness of different lines of VTE prophylaxis after major orthopedic procedures.

3- Studies that compared those interventions with each other or no comparison.

4- Studies that reported any of the following outcomes:

\section{Postoperative VTE.}

Pulmonary embolism (PE)

Bleeding

Major adverse event.

In-hospital mortality.

5- Studies that were randomized controlled trials (RCTs), comparative studies, or prospective cohort studies.

6- Studies that were published since 2010 to November 2019.

We excluded review articles, non-English studies, theses, dissertations and conference abstracts, and trials with unreliable date for extraction.

An electronic search was conducted from 2010 till November 2019 in the following bibliographic databases: Medline via 
PubMed, SCOPUS, Cochrane Central Register of Controlled Trials (CENTRAL), and Web of Science to identify relevant articles. We used different combinations of the following queries: ((("Arthroplasty, Replacement, Hip"[Mesh]) OR "Arthroplasty, Replacement, Knee"[Mesh]) OR "Hip Fractures"[Mesh]) AND "Venous Thromboembolism"[Mesh]. Also we used other keywords like venous thromboembolism after orthopedic surgeries, knee arthroscopy, pharmacological and mechanical prophylaxis and guidelines for DVT prophylaxis.

\section{Results}

In the present study, we searched Medline via PubMed, SCOPUS, Web of Science, Cochrane Central Register of Controlled Trials (CENTRAL), and Google Scholar from 2010 till December 2019. The search retrieved 2089 unique records. We then retained 57 potentially eligible records for full-texts screening. Finally, 29 studies were included (Figure 1).

Overall, twelve studies reported the rates of DVT among patients receiving LMWH. The overall effect estimates showed that the rate of DVT was $9.2 \%$ (95\% CI 6.6- 11.8\%). The pooled studies showed significant heterogeneity ( $p=0.001 ; I^{2}=83 \%$; Figure. 2 ).

Overall, nine studies reported the rates of DVT among patients receiving FXaI. The overall effect estimates showed that the rate of DVT was $3.9 \%$ (95\% CI 1.9-6\%). The pooled studies showed significant heterogeneity ( $p=0.001 ; \mathrm{I}^{2}=85 \%$; Figure. 3 ).

Nine studies compared LMWH versus FXaI for the rates of DVT. The overall effect estimates favored FXaI over LMWH (RR $0.42,95 \%$ CI $[0.19-0.96], \mathrm{P}=0.04)$. The pooled studies showed significant heterogeneity ( $p=0.001 ; \mathrm{I}^{2}=86 \%$; Figure.4).

Nine studies reported the rates of PE among patients receiving LMWH. The overall effect estimates showed that the rate of PE was $0.3 \%$ (95\% CI $0.1-0.6 \%)$. The pooled studies showed no significant heterogeneity ( $p=0.84 ; I^{2}=0 \% ;$ Figure .5 ).

Six studies reported the rates of PE among patients receiving FXaI. The overall effect estimates showed that the rate of PE was $0.6 \%$ (95\% CI $0.02-1 \%$ ). The pooled studies showed no significant heterogeneity $(\mathrm{p}=0.97$; $\mathrm{I}^{2}=0 \%$; Figure.6).

Six studies compared LMWH versus FXaI for the rates of PE. The overall effect estimates did not favor any of the two drugs (RR $0.43,95 \%$ CI [0.24 - 1.9], $\mathrm{P}=0.28$ ). The pooled studies showed no significant heterogeneity ( $p=0.702 ; I^{2}=0 \%$; Figure. 7$)$.

Ten studies reported the rates of major bleeding among patients receiving LMWH. The overall effect estimates showed that the 
rate of major bleeding was $1.3 \%$ (95\% CI 0.6-1.9\%). The pooled studies showed significant heterogeneity $\left(\mathrm{p}=0.002 ; \mathrm{I}^{2}=66 \%\right.$; Figure.8)

Seven studies reported the rates of major bleeding among patients receiving FXaI. The overall effect estimates showed that the rate of major bleeding was $0.4 \%$ (95\% CI $0.02-$ $0.7 \%)$. The pooled studies showed no significant heterogeneity $\left(\mathrm{p}=0.59 ; \mathrm{I}^{2}=0 \%\right.$; Figure.9).

Six studies compared LMWH versus FXaI for the rates of major bleeding. The overall effect estimates favored FXaI over LMWH (RR 0.34, 95\% CI [0.17 - 0.73], $\mathrm{P}=0.28$ ). The pooled studies showed no significant heterogeneity $\left(\mathrm{p}=0.78 ; \mathrm{I}^{2}=0 \%\right.$; Figure. 10 )

Overall, five studies reported the rates of proximal DVT among patients receiving LMWH. The overall effect estimates showed that the rate of proximal DVT was $1.4 \%$ (95\% CI $0.6-2.3 \%$ ). The pooled studies showed no significant heterogeneity $(\mathrm{p}=0.29$; $\mathrm{I}^{2}=18 \%$; Figure. 11 ).

Three studies reported the rates of symptomatic DVT among patients receiving LMWH. The overall effect estimates showed that the rate of symptomatic DVT 1\% (95\% CI $0.1-1.8 \%$ ). The pooled studies showed no significant heterogeneity ( $\mathrm{p}=0.19 ; \mathrm{I}^{2}=39 \%$; Figure.12).
Six studies reported the rates of total DVT among patients receiving LMWH. The overall effect estimates showed that the rate of total DVT $11.2 \%$ (95\% CI 3.5- 18.8\%). The pooled studies showed significant heterogeneity ( $\mathrm{p}=0.001 ; \mathrm{I}^{2}=93 \%$; Figure.13).

Overall, two studies reported the rates of symptomatic DVT among patients receiving FXaI. The overall effect estimates showed that the rate of symptomatic DVT was $0.5 \%$ (95\% CI $0.3-1.4 \%)$. The pooled studies showed no significant heterogeneity $(\mathrm{p}=0.75$; $\mathrm{I}^{2}=0 \%$; Figure.14).

Four studies reported the rates of major bleeding among patients receiving LMWH. The overall effect estimates showed that the rate of major bleeding was $0.5 \%(95 \% \mathrm{CI}$ $0.1-1 \%)$. The pooled studies showed no significant heterogeneity $\left(\mathrm{p}=0.57 ; \mathrm{I}^{2}=0 \%\right.$; Figure.15).

Overall, four studies reported the rates of symptomatic VTE among patients receiving prophylaxis. The overall effect estimates showed that the rate of symptomatic VTE was $0.7 \%$ (95\% CI $0.2-1.1 \%$ ). The pooled studies showed no significant heterogeneity ( $p=0.91 ; I^{2}=0 \%$; Figure. 16$)$.

Four studies reported the rates of symptomatic and asymptomatic VTE among patients receiving prophylaxis. The overall effect estimates showed that the rate of 
symptomatic and asymptomatic VTE was studies showed no significant heterogeneity $\left(\mathrm{p}=0.82 ; \mathrm{I}^{2}=0 \%\right.$; Figure. 17$)$.

Overall, four studies reported the rates of major bleeding among patients receiving prophylaxis. The overall effect estimates showed that the rate of major bleeding was $0.2 \%(95 \%$ CI $0-0.4 \%)$. The pooled studies showed no significant heterogeneity $(\mathrm{p}=0.75$; $\mathrm{I}^{2}=0 \%$; Figure.18).
$0.8 \% \quad(95 \%$ CI $0.2-1.3 \%)$. The pooled Overall, two studies reported the rates of all bleeding among patients receiving prophylaxis. The overall effect estimates showed that the rate of major bleeding was $6.6 \%$ (95\% CI 4-12.9\%). The pooled studies showed significant heterogeneity $(\mathrm{p}=0.001$; $\mathrm{I}^{2}=90 \%$; Figure19

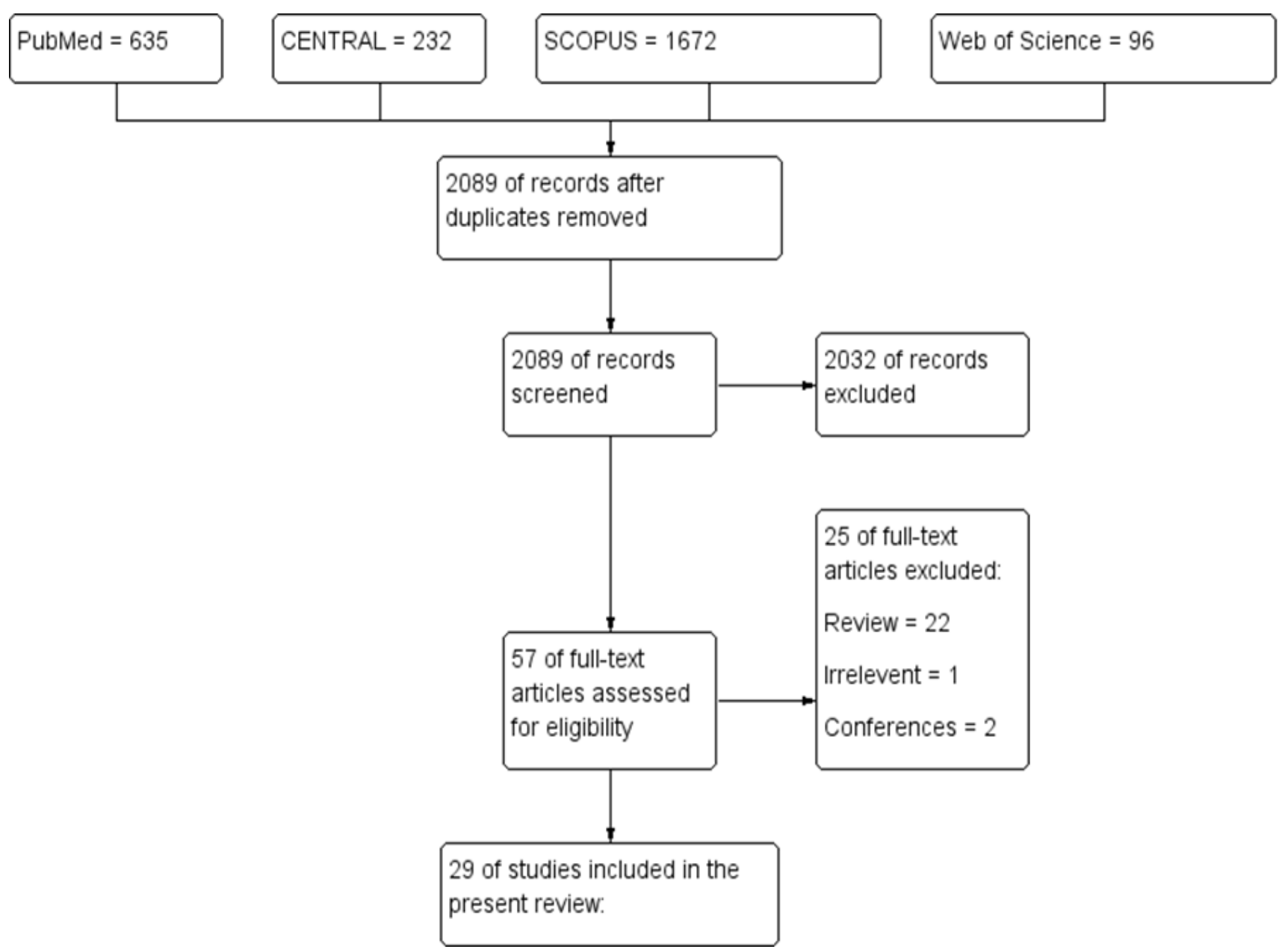

Figure 1: PRISMA flow-chart 


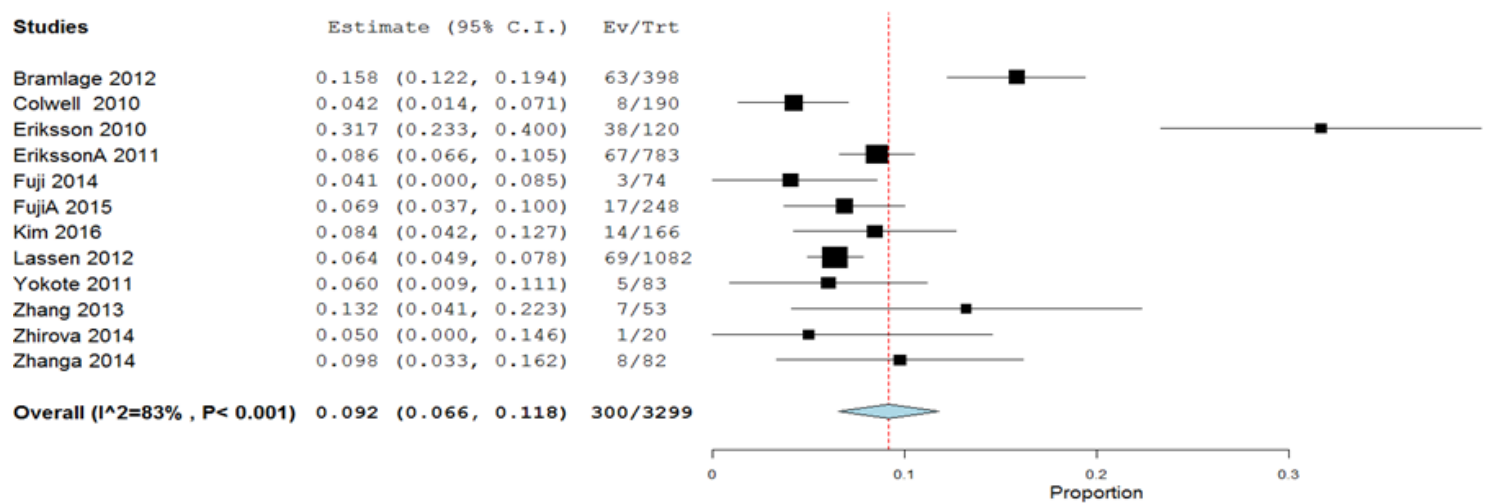

Figure 2: Forest Plot of rates of DVT among patients receiving LMWH after THR

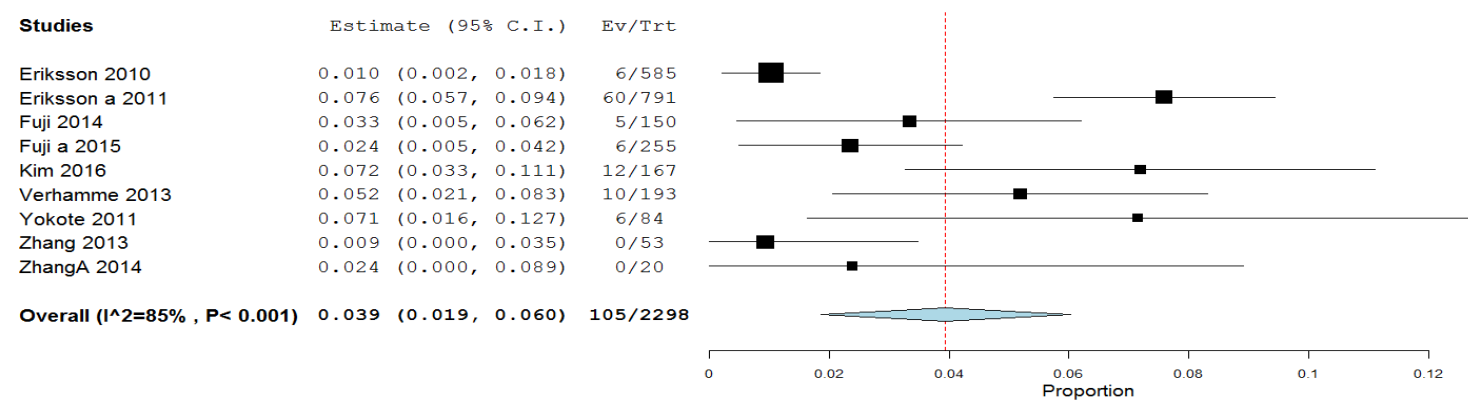

Figure 3: Forest Plot of rates of DVT among patients receiving FXaI after THR

Studies

Eriksson 2010 Eriksson a 2011 Fuji 2014

Fuji a 2015

Kim 2016

Verhamme 2013

Yokote 2011

Zhang 2013

ZhangA 2014

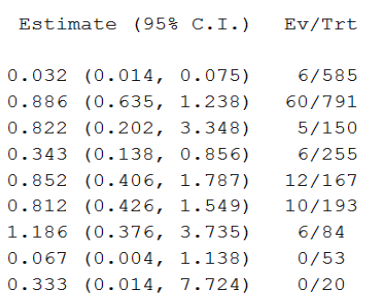

Ev/Ctrl

$38 / 120$

$38 / 120$
$67 / 783$

$3 / 74$

$17 / 248$

$14 / 166$

$69 / 1082$

$5 / 83$

$7 / 53$

$1 / 20$

Overall $\left(\left.\right|^{\wedge} 2=86 \%, P<0.001\right) \quad 0.428 \quad(0.191,0.961) \quad 105 / 2298 \quad 221 / 2629$

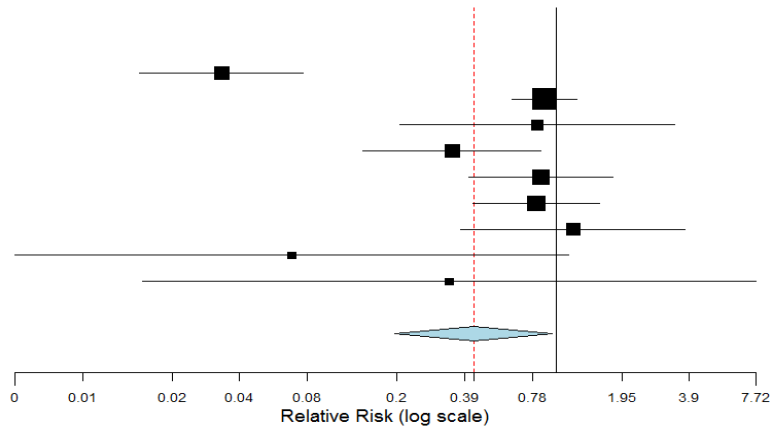

Figure 4: Forest Plot of rates of DVT for LMWH versus FXaI after THR

Studies

Andersson 2013

Borgen 2012

Bramlage 2012

Colwell 2010

Eriksson 2010

Eriksson A 2011

Fuji 2014

Fuji A 2015

Kim 2016

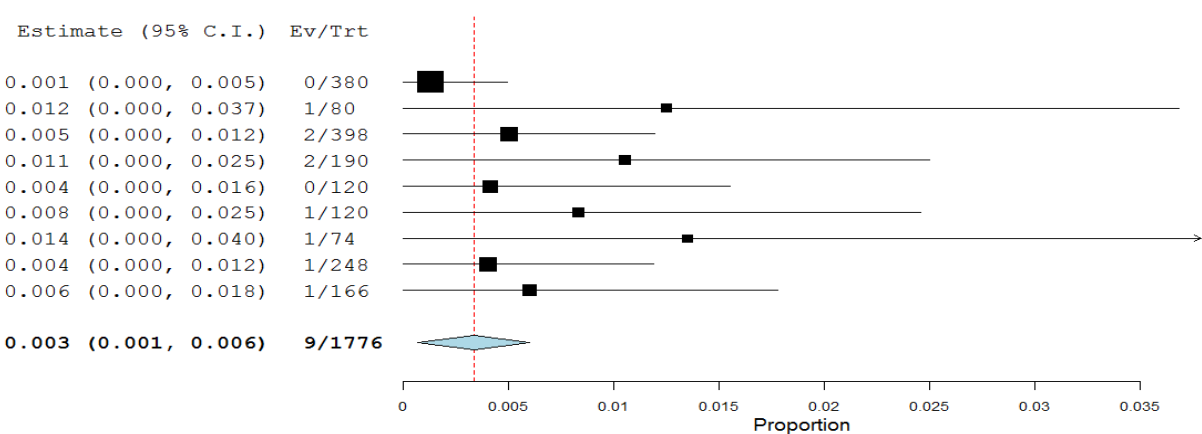

Figure 5: Forest Plot of rates of PE among patients receiving LMWH after THR 


\begin{tabular}{|c|c|c|c|}
\hline Studies & Estimate $195 \%$ & C.I.) & $\mathrm{Ev} / \operatorname{Tr} \mathrm{t}$ \\
\hline Eriksson 2010 & $0.009 \quad(0.001$, & $0.016)$ & $5 / 585$ \\
\hline Eriksson a 2011 & $0.009 \quad(0.000$ & $0.026)$ & $1 / 114$ \\
\hline Fuji 2014 & $0.007 \quad 0.000$, & $0.020)$ & $1 / 150$ \\
\hline Fuji a 2015 & $0.004 \quad(0.000$, & $0.012)$ & $1 / 255$ \\
\hline Kim 2016 & $0.006 \quad 0.000$ & $0.018)$ & $1 / 167$ \\
\hline Verhamme 2013 & $0.005(0.000$, & $0.015)$ & $1 / 191$ \\
\hline
\end{tabular}

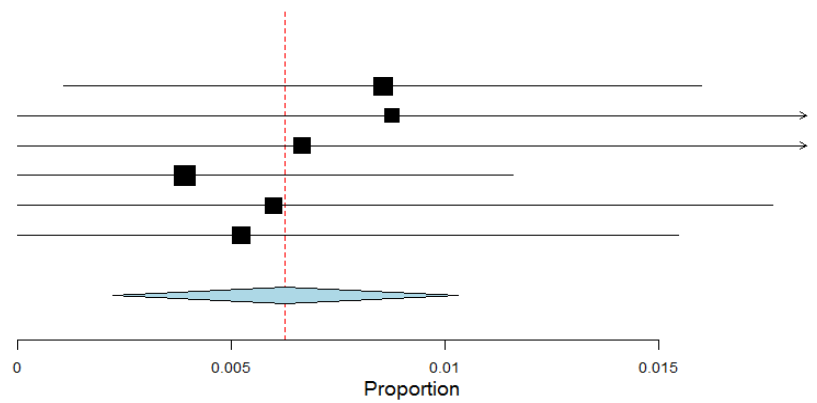

Figure 6: Forest Plot of rates of PE among patients receiving FXaI after THR

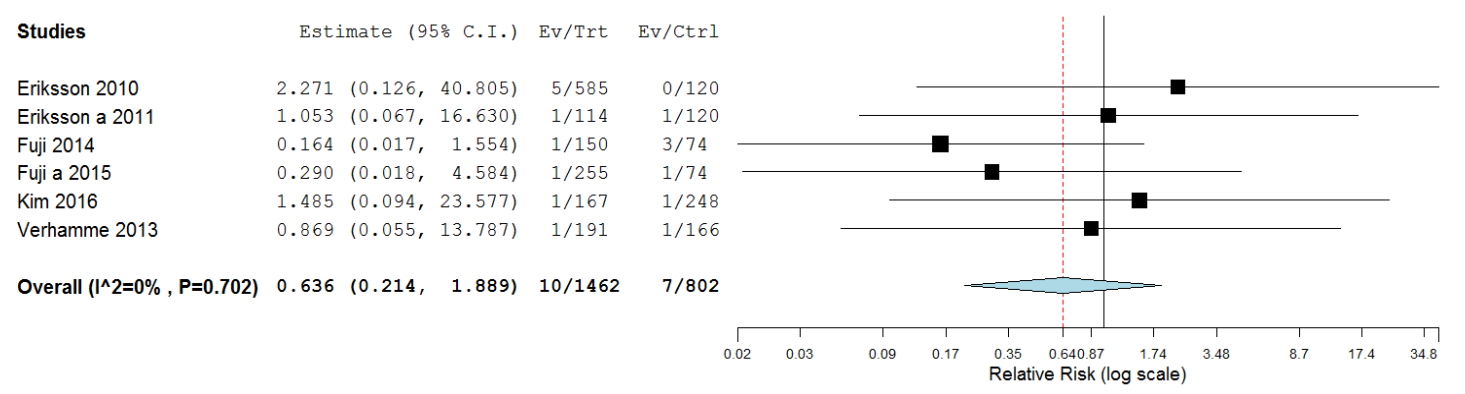

Figure 7: Forest Plot of rates of PE for LMWH versus FXaI after THR

Studies
Andersson 2013
Borgen 2012
Colwell 2010
Eriksson 2010
Eriksson A 2011
Eriksson B 2014
Fuji 2014
Fuji A 2015
Lassen 2012
Yokote 2011
Overall (|^2 $\mathbf{2}=\mathbf{6 6 \%}, \mathbf{P = 0 . 0 0 2 )}$

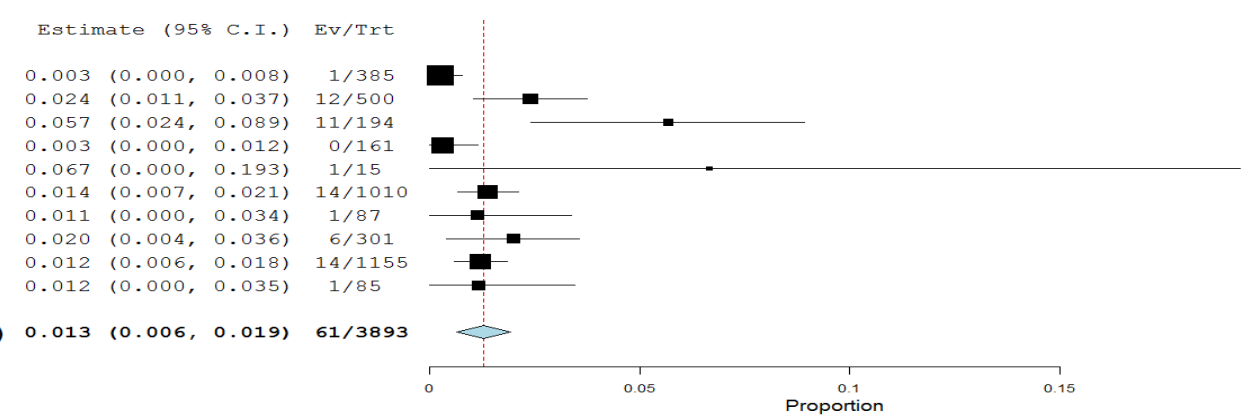

Figure 8: Forest Plot of rates of major bleeding among patients receiving LMWH after THR

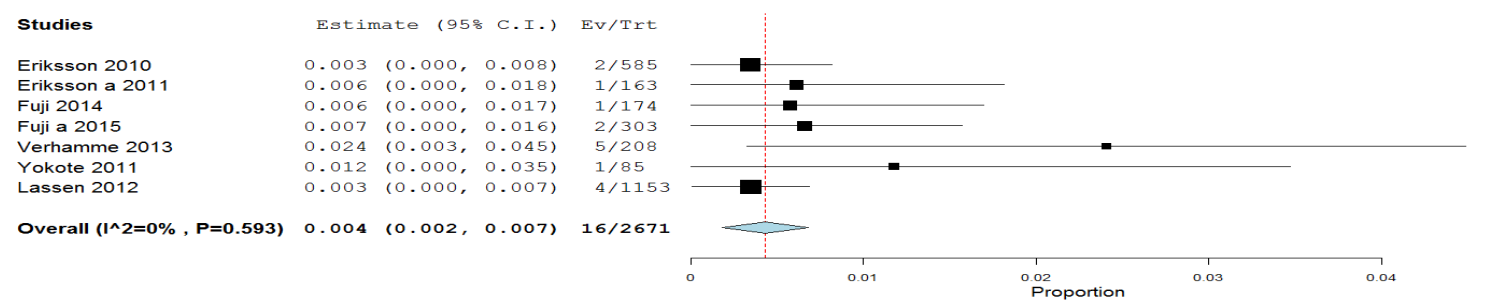

Figure 9: Forest Plot of rates of major bleeding among patients receiving FXaI after THR

\begin{tabular}{|c|c|c|c|c|}
\hline Studies & Estimate & 95\% C.I.) & $\mathrm{Ev} / \operatorname{Trt}$ & $\mathrm{Ev} / \mathrm{Ctrl}$ \\
\hline Eriksson 2010 & $1.382(0.067$ & $28.648)$ & $2 / 585$ & $0 / 161$ \\
\hline Eriksson a 2011 & $0.092(0.006$ & $1.398)$ & $1 / 163$ & $1 / 15$ \\
\hline Fuji 2014 & $0.500 \quad(0.032$ & 7.899) & $1 / 174$ & $1 / 87$ \\
\hline Fuji a 2015 & $0.331(0.067$ & $1.628)$ & $2 / 303$ & $6 / 301$ \\
\hline Yokote 2011 & $1.000(0.064$, & $15.728)$ & $1 / 85$ & $1 / 85$ \\
\hline Lassen 2012 & $0.286(0.094$, & $0.867)$ & $4 / 1153$ & $14 / 1155$ \\
\hline
\end{tabular}

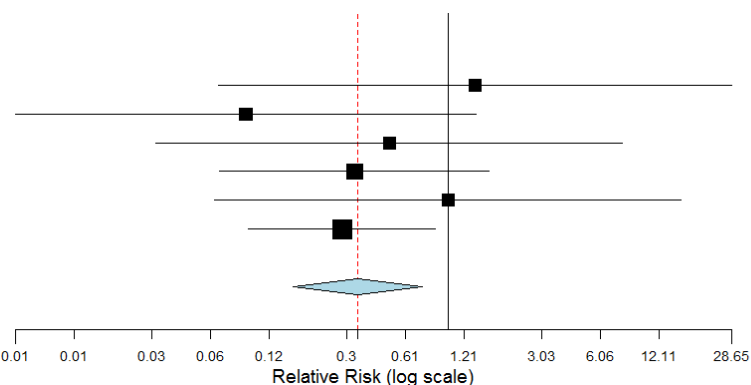


Figure 10: Forest Plot of rates of major bleeding for LMWH versus FXaI after THR

Studies

Barrellier 2010 Barrellier 2010 (b)

Jiang 2014

Mirdamadi 201

Büller 2015 (b)
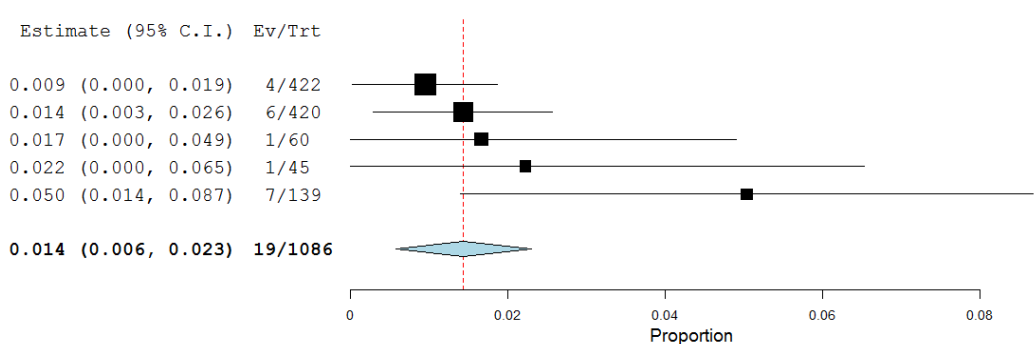

Figure 11: Forest Plot of rates of proximal DVT among patients receiving LMWH after TKR

Studies

Barrellier 2010 Barrellier 2010 (b) Büller 2015 (b)

Estimate (95\% C.I.) Ev/Trt

$0.005(0.000,0.011) \quad 2 / 422$ $0.017(0.004,0.029) \quad 7 / 420$ $0.014(0.000,0.034) \quad 2 / 139$

Overall $\left(\left.\right|^{\wedge} 2=39 \%, P=0.194\right) \quad 0.010(0.001,0.018) \quad 11 / 981$
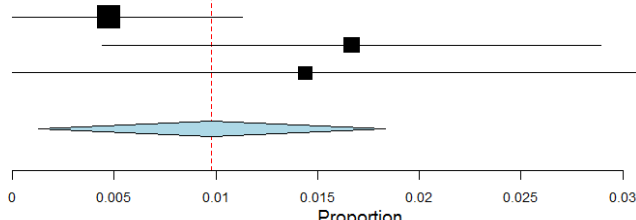

Figure 12: Forest Plot of rates of symptomatic DVT among patients receiving LMWH after TKR

Studies

Barrellier 2010 Barrellier 2010 (b)

Hu 2015

Jiang 2014

Mirdamadi 2014

Büller 2015 (b)

Overall (|^2=93\%, P< 0.001) $0.112 \quad(0.035,0.188) \quad 66 / 431$

Estimate (95\% C.I.) Ev/Trt

$0.042(0.000,0.089) \quad 3 / 71$

$0.310(0.202,0.417) 22 / 71$

$0.089(0.006,0.172) \quad 4 / 45$

$0.008(0.000,0.031) \quad 0 / 60$

$0.022(0.000,0.065) \quad 1 / 45$

$0.259(0.186,0.332) \quad 36 / 139$

年

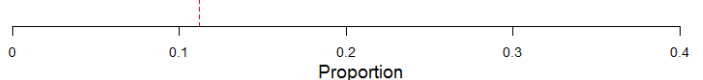

Figure 13: Forest Plot of rates of total DVT among patients receiving LMWH after TKR

Studies

Estimate (95\% C.I.) Ev/Trt

Cohen 2013

Büller 2015

$0.004 \quad(0.000,0.016) \quad 0 / 120$

$0.007(0.000,0.021) \quad 1 / 142$

Overall $\left(I^{\wedge} 2=0 \%, P=0.750\right) \quad 0.005 \quad(-0.003,0.014) \quad 1 / 208$

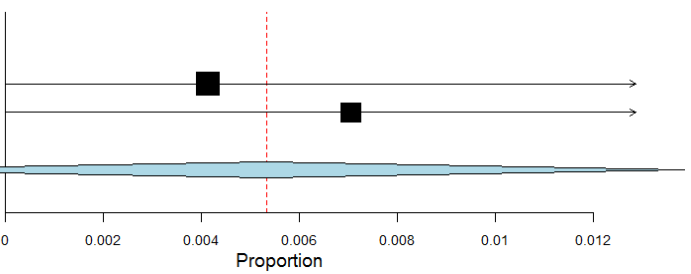

Figure 24: Forest Plot of rates of symptomatic DVT among patients receiving FXaI after TKR

Studies

Estimate (95\% C.I.) Ev/Trt

Barrellier 2010

Barrellier 2010 (b)

Mirdamadi 2014

$0.007(0.000,0.015) \quad 3 / 422$

$0.005(0.000,0.011) \quad 2 / 420$

$0.044(0.000,0.105) \quad 2 / 45$

$0.003(0.000,0.013) \quad 0 / 144$

Overall (l^2=0\%, P=0.575) $0.005 \quad(0.001,0.010) \quad 7 / 1031$

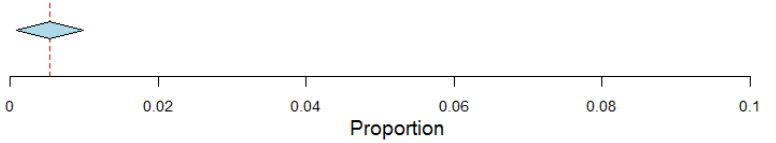

Figure 15: Forest Plot of rates of major bleeding among patients receiving LMWH after TKR 


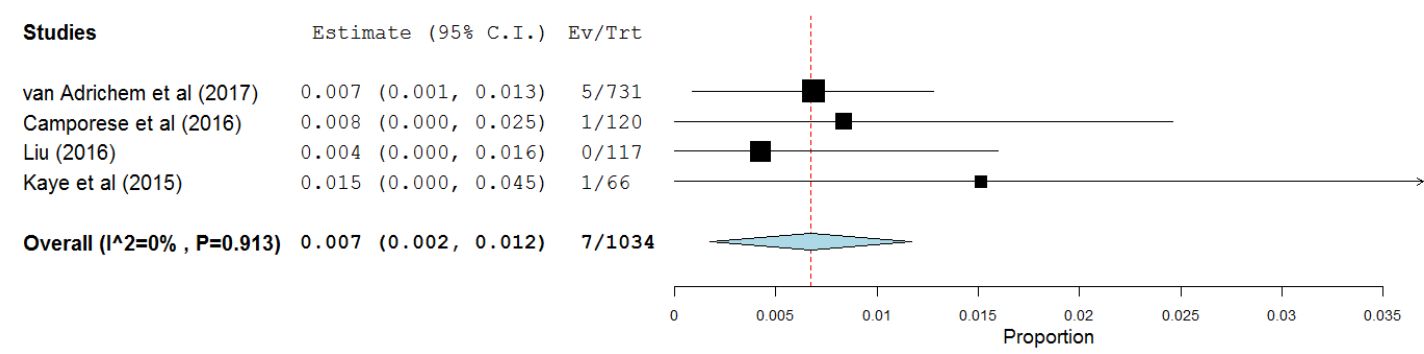

Figure 16: Forest Plot of rates of symptomatic VTE among patients receiving prophylaxis after arthroscopic knee surgery
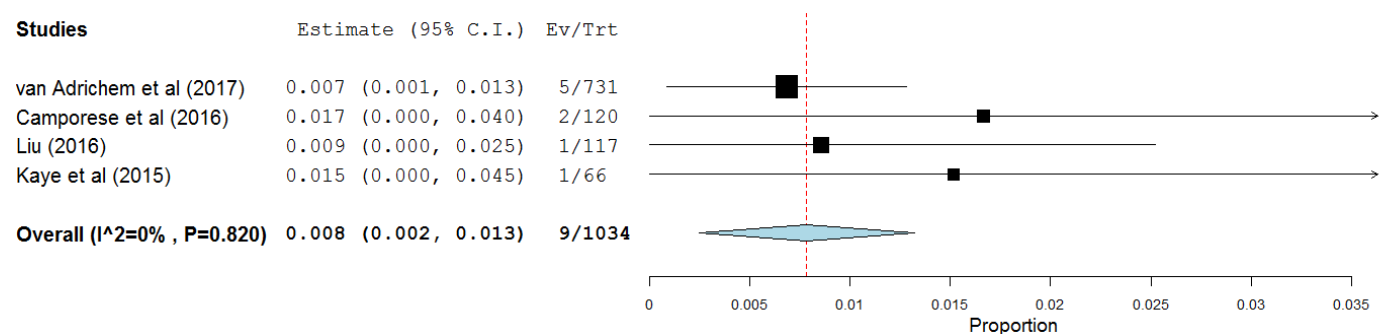

Figure 17: Forest Plot of rates of symptomatic and asymptomatic VTE after arthroscopic knee surgery

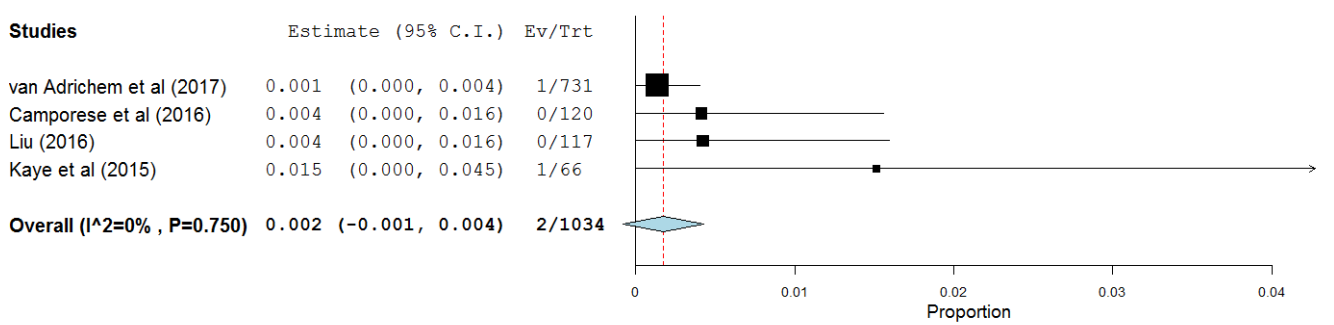

Figure 18: Forest Plot of rates of major bleeding events among patients after arthroscopic knee surgery

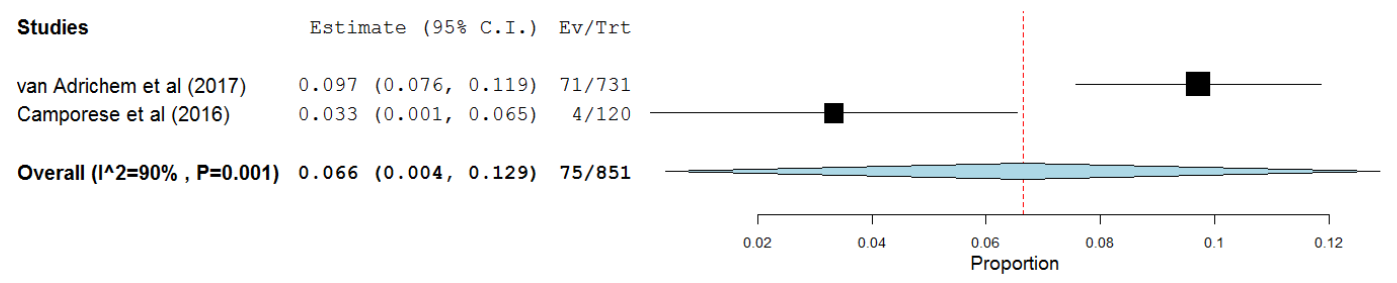

Figure 19: Forest Plot of rates of all bleeding after arthroscopic knee surgery

\section{Discussion}

RCTs comparing LMWH with placebo in patients who receive THR or TKR are decreasing recently which is expected as there has been an established evidence on the need for VTE thromboprophylaxis. However, the efficacy and safety of different agent became the center of the research. An old systematic review (9), assessed the use of LMWH compared with placebo in the prevention of thrombosis in an out-patient setting in patients undergoing hip arthroplasty. Compared to placebo, LMWH was associated with decreased rates of DVT, 
proximal venous thrombosis, and symptomatic venous thrombosis.

Similarly Tasker et al (10), assessed the clinical outcomes of LMWH compared with placebo in patients who had THA. The results of their meta-analysis showed similarity in the risk of pulmonary embolism, all-cause mortality or major bleeding. However, compared with placebo, LMWH reduced incidence of non-fatal PEs but at the expense of hematoma formation.

And as stated in our review's results regarding the superiority of FXaI over LMWH in thromboembolic prophylaxis after THR and TKR with more bleeding complications occurring with LMWH, yet our review did not compare between different agents of FXaIs, previous meta-analyses found that the factor $\mathrm{Xa}$ inhibitors rivaroxaban and apixaban had a better anticoagulant effect compared with enoxaparin. They also found that enoxaparin had a higher incidence of major bleeding compared with some, but not all, of the factor Xa inhibitors. For example, Gomez-Outes et al (11) found that compared with enoxaparin, the risk of clinically relevant bleeding was higher with rivaroxaban, similar with dabigatran, and lower with apixaban in patients having THR or TKR. The authors concluded that the higher efficacy with factor Xa inhibitors was generally associated with a higher bleeding tendency compared with enoxaparin.

Also in agreement to our review, oral FXaI were more effective than LMWH in venous thromboembolic prophylaxis with lower risk of bleeding after THR and TKR. A metaanalysis conducted by Feng et al (12), they analyzed RCTs which compared the efficacy of oral direct factor Xa inhibitor with that of LMWH specially enoxaparin for elective TKR or THR. The direct factor Xa inhibitor included rivaroxaban, apixaban, edoxaban, and several developing drugs. The study concluded that oral FXaIs are more effective to prevent thromboembolic events after THR or TKR. Rivaroxaban, apixaban and edoxaban showed a better anticoagulant effect as compared with enoxaparin. The study also found that rivaroxaban was associated with a higher bleeding incidence than enoxaparin, while apixaban and edoxaban were not associated with significantly higher bleeding risks.

\section{Conclusion}

As per our systematic review and metaanalysis comparing between different groups of anticoagulants in thromboembolic prophylaxis following THR, TKR, hip fracture and knee arthroscopic surgeries, FXaI was the most effective agent after THR and TKR. In hip fracture surgery and Knee arthroscopy, thromboprophylaxis is needed, 
but variable results regarding the drug choice warrant more research.

\section{References}

1. Kakkar AK, Cohen AT, Tapson VF, Bergmann JF, Goldhaber SZ, Deslandes B, et al. Venous thromboembolism risk and prophylaxis in the acute care hospital setting (ENDORSE survey): findings in surgical patients. Ann Surg. 2010;251(2):330-8.

2. Prandoni P, Noventa F, Ghirarduzzi A, Pengo V, Bernardi E, Pesavento R, et al. The risk of recurrent venous thromboembolism after discontinuing anticoagulation in patients with acute proximal deep vein thrombosis or pulmonary embolism. A prospective cohort study in 1,626 patients. Haematologica. 2007;92(2):199-205.

3. Geerts WH, Pineo GF, Heit JA, Bergqvist D, Lassen MR, Colwell CW, et al. Prevention of venous thromboembolism: the Seventh ACCP Conference on Antithrombotic and Thrombolytic Therapy. Chest. 2004;126(3):338S-400S.

4. Næss IA, Christiansen SC, Romundstad P, Cannegieter SC, Rosendaal FR, Hammerstrøm J. Incidence and mortality of venous thrombosis: a population-based study. J Thromb Haemost. 2007;5(4):692-9.

5. Parasuraman S, Goldhaber SZ. Venous thromboembolism in children. Circulation. 2006;113(2):e12-6.

6. Hirsh J, Guyatt G, Albers GW, Harrington R, Schünemann HJ. Antithrombotic and thrombolytic therapy: American College of Chest Physicians evidence-based clinical practice guidelines. Chest. 2008;133(6):110S-112S.

7. Yu H-T, Dylan ML, Lin J, Dubois RW. Hospitals' compliance with prophylaxis guidelines for venous thromboembolism. Am J Heal Pharm.
2007;64(1):69-76.

8. Moher D, Liberati A TJ and AD. The PRISMA Group. Preferred Reporting Items for Systematic Reviews and Meta-Analyses: The PRISMA Statement. Ann Intern Med. 2009;151(4):264-9.

9. Hull RD, Pineo GF, Stein PD, Mah AF, MacIsaac SM, Dahl OE, et al. Extended out-of-hospital lowmolecular-weight heparin prophylaxis against deep venous thrombosis in patients after elective hip arthroplasty: a systematic review. Ann Intern Med. 2001;135(10):858-69.

10. Tasker A, Harbord R, Bannister G. Metaanalysis of low molecular weight heparin versus placebo in patients undergoing total hip replacement and post-operative morbidity and mortality since their introduction. Hip Int. 2010;20(1):64-74.

11. Gómez-Outes A, Terleira-Fernández AI, Suárez-Gea ML, Vargas-Castrillón E. Dabigatran, rivaroxaban, or apixaban versus enoxaparin for thromboprophylaxis after total hip or knee replacement: systematic review, meta-analysis, and indirect treatment comparisons. Bmj. 2012;344:e3675.

12. Feng $\mathrm{W}, \mathrm{Wu} \mathrm{K}$, Liu Z, Kong G, Deng Z, Chen $\mathrm{S}$, et al. Oral direct factor Xa inhibitor versus enoxaparin for thromboprophylaxis after hip or knee arthroplasty: systemic review, traditional metaanalysis, dose-response meta-analysis and network meta-analysis. Thromb Res. 2015;136(6):1133-44.

13. Huisman M V, Quinlan DJ, Dahl OE, Schulman S. Enoxaparin versus dabigatran or rivaroxaban for thromboprophylaxis after hip or knee arthroplasty: results of separate pooled analyses of phase III multicenter randomized trials. Circ Cardiovasc Qual Outcomes. 2010;3(6):652-60.

14. Wolowacz SE, Roskell NS, Plumb JM, Caprini JA, Eriksson BI. Efficacy and safety of 
dabigatran etexilate for the prevention of venous thromboembolism following total hip or knee arthroplasty. Thromb Haemost. 2009;101(01):7785.

15. Cohen AT, Hirst C, Sherrill B, Holmes P, Fidan D. Meta-analysis of trials comparing ximelagatran with low molecular weight heparin for prevention of venous thromboembolism after major orthopaedic surgery. Br J Surg. 2005;92(11):133544.

16. Colleoni JL, Ribeiro FN, Mos PAC, Reis JP, de Oliveira HR, Miura BK. Venous thromboembolism prophylaxis after total knee arthroplasty (TKA): aspirin vs. rivaroxaban. Rev Bras Ortop (English Ed. 2018;53(1):22-7.
17. Anderson DR, Dunbar M, Murnaghan J, Kahn SR, Gross P, Forsythe M, et al. Aspirin or rivaroxaban for VTE prophylaxis after hip or knee arthroplasty. N Engl J Med. 2018;378(8):699-707.

18. Weitz JI, Lensing AWA, Prins MH, Bauersachs R, Beyer-Westendorf J, Bounameaux H, et al. Rivaroxaban or aspirin for extended treatment of venous thromboembolism. $\mathrm{N}$ Engl $\mathrm{J}$ Med. 2017;376(13):1211-22.

19. Yu Y, Lu S, Sun J, Zhou W, Liu H. Thromboprophylactic Efficacy and Safety of Anticoagulants After Arthroscopic Knee Surgery: A Systematic Review and Meta-Analysis. Clin Appl Thromb. 2019;25:1076029619881409.

To cite this article: Sheren A. Khalil, Ahmed S. Rizk, Ahmed A. Elsheikh, Bishoy N. Ragheb. Methods of Venous Thromboembolic Prophylaxis in Orthopedic Surgeries, BMFJ 2020;37(3):710-722, DOI: 10.21608/bmfj.2020.31740.1272 